PROCEEDINGS OF THE

AMERICAN MATHEMATICAL SOCIETY

Volume 135, Number 9, September 2007, Pages 2887-2891

S 0002-9939(07)08802-8

Article electronically published on May 8, 2007

\title{
FROM AN ITERATION FORMULA TO POINCARÉ'S ISOCHRONOUS CENTER THEOREM FOR HOLOMORPHIC VECTOR FIELDS
}

\author{
GUANG YUAN ZHANG \\ (Communicated by Carmen C. Chicone)
}

\begin{abstract}
We first generalize a classical iteration formula for one variable holomorphic mappings to a formula for higher dimensional holomorphic mappings. Then, as an application, we give a short and intuitive proof of a classical theorem, due to H. Poincaré, for the condition under which a singularity of a holomorphic vector field is an isochronous center.
\end{abstract}

\section{An iteration Formula}

Let $f$ be a holomorphic function germ at the origin in the complex plane with $f(0)=0$. For each positive integer $k$, we denote by $f^{k}$ the $k$-th iteration of $f$ defined as $f^{1}=f, f^{2}=f \circ f, \ldots, f^{k}=f \circ f^{k-1}$ inductively, which is a well defined holomorphic function germ at the origin.

Assume that $\lambda=f^{\prime}(0)$ is a primitive $m$-th root of unity, say, $\lambda^{m}=1$, but $\lambda^{j} \neq 1$ for each positive integer $j$ with $j<m$. Then it is interesting that there exists a positive integer $r$, such that the $m$-th iteration $f^{m}$ has a power series expansion

$$
f^{m}(z)=z+a_{1} z^{r m+1}+a_{2} z^{r m+2}+\ldots
$$

at the origin: all the terms of degrees from 2 to $\mathrm{rm}$ vanish! This can be proved by applying Rouché's theorem (see 12]). In this section we generalize this formula to germs of higher dimensional mappings by using normal form theory. We denote by $\mathbb{C}^{n}$ the complex vector space and by $\Delta^{n}$ a ball in $\mathbb{C}^{n}$ centered at the origin.

Proposition 1 (Iteration Formula). Let $f: \Delta^{n} \rightarrow \mathbb{C}^{n}$ be a holomorphic mapping given by

$$
f(z)=\lambda z+o(|z|), \quad z \in \Delta^{n},
$$

where $\lambda$ is a primitive $m$-th root of unity. Then, in a neighborhood of the origin,

$$
f^{m}(z)=z+o\left(|z|^{m}\right) \text {. }
$$

In the proposition, $z=\left(z_{1}, \ldots, z_{n}\right)$ and the expression $o\left(|z|^{m}\right)$ means that each component of the mapping $f^{m}(z)-z$ is a power series in $z_{1}, \ldots, z_{n}$ consisting of terms of degree $>m$.

Received by the editors November 23, 2005 and, in revised form, May 30, 2006

2000 Mathematics Subject Classification. Primary 32H50, 32M25, 37C27.

Key words and phrases. Ordinary differential equation, holomorphic differential equation.

The author is supported by Chinese NSFC 10271063 and 10571009.

(C)2007 American Mathematical Society Reverts to public domain 28 years from publication 
Proof. By the hypothesis and a fundamental result in the normal form theory (see [1] or pages $84-85$ in [2] for the proof), there exists a biholomorphic transformation in the form of

$$
z=\left(z_{1}, \ldots, z_{n}\right)=h\left(x_{1}, \ldots, x_{n}\right)=\left(x_{1}, \ldots, x_{n}\right)+o(|x|)
$$

of coordinates in a neighborhood of the origin such that each component $g_{j}$ of $g=h^{-1} \circ f \circ h=\left(g_{1}, \ldots, g_{n}\right)$ has a power series expansion

$$
g_{j}\left(x_{1}, \ldots, x_{n}\right)=\lambda x_{j}+\sum c_{i_{1} \ldots i_{n}}^{j} x_{1}^{i_{1}} \ldots x_{n}^{i_{n}}+o\left(|x|^{m}\right), \quad j=1, \ldots, n,
$$

in a neighborhood of the origin, where $x=\left(x_{1}, \ldots, x_{n}\right)$ and the sum extends over all $n$-tuples $\left(i_{1}, \ldots, i_{n}\right)$ of nonnegative integers with

$$
2 \leq i_{1}+\cdots+i_{n} \leq m \text { and } \lambda^{i_{1}+\cdots+i_{n}}=\lambda .
$$

On the other hand, since $\lambda$ is a primitive $m$-th root of unity, we have

$$
\lambda^{i_{1}+\cdots+i_{n}} \neq \lambda \text { if } 2 \leq i_{1}+\cdots+i_{n} \leq m .
$$

Therefore, the sum in the equation (1.3) vanishes, and then we have

$$
g(x)=\lambda x+o\left(|x|^{m}\right)
$$

and then, considering that $\lambda^{m}=1$, we conclude that the $m$-th iteration $g^{m}$ can be expressed as

$$
g^{m}(x)=x+o\left(|x|^{m}\right) .
$$

By (1.2) it is clear that $o\left(\left|h^{-1}(z)\right|^{m}\right)=o\left(|z|^{m}\right)$, and then, by (1.4) it is easy to see that

$$
\begin{aligned}
& f^{m}(z)=h \circ g^{m} \circ h^{-1}(z)=h\left(h^{-1}(z)+o\left(\left|h^{-1}(z)\right|^{m}\right)\right) \\
= & h\left(h^{-1}(z)+o\left(|z|^{m}\right)\right)=h\left(h^{-1}(z)\right)+o\left(|z|^{m}\right) \\
= & z+o\left(|z|^{m}\right) .
\end{aligned}
$$

This completes the proof.

\section{Poincaré's CONDITION FOR ISOChronous CENTERS}

Consider an $n$-dimensional complex holomorphic system

$$
\dot{z}=F(z), \quad z \in \Delta^{n},
$$

such that the origin $z=0$ is a singularity. This means that $F$ is a holomorphic mapping from $\Delta^{n}$ into $\mathbb{C}^{n}$ such that $F(0)=0$.

The origin is called a center of the system if it has a punctured neighborhood that is filled with periodic orbits, and called an isochronous center if it has a punctured neighborhood that is filled with periodic orbits of the same period. Here, and throughout this paper, the period of a periodic orbit means the smallest positive one.

The problem to find the condition so that a singularity of a system is a center has a long history. The term center was defined by H. Poincaré, while the research of center phenomena were started in 1673 when Huygens studied the cycloidal pendulum (see [7]). As an application of the previous proposition, we shall present a short and intuitive proof of the following classical theorem due to H. Poincaré. 
Isochronous Center Theorem. If the Jacobian matrix $F^{\prime}(0)$ of $F$ at the origin equals wiI for some real number $\omega \neq 0$, where $i=\sqrt{-1}$ and $I$ is the unit matrix, then the origin is an isochronous center with period $2 \pi /|\omega|$.

This result follows from Poincaré's linearization theorem, which asserts that the system (2.1) is linearizable at the origin via a biholomorphic transformation of coordinates, provided that the $n$-tuple $\left(\lambda_{1}, \ldots, \lambda_{n}\right)$ of all eigenvalues of $F^{\prime}(0)$ is in the Poincaré domain: the convex hull of these eigenvalues in the complex plane does not contain the origin, and that there is no resonance: for any $n$-tuple $\left(i_{1}, \ldots, i_{n}\right)$ of nonnegative integers with $i_{1}+\cdots+i_{n} \geq 2, \lambda_{j} \neq \lambda_{1}^{i_{1}} \ldots \lambda_{n}^{i_{n}}$ for each $j=1,2, \ldots, n$ (see Chapter 5 in [1] for the details).

It is interesting that in the history of the study of central singularities, special cases of the Isochronous Center Theorem have been rediscovered several times. For example, when $n=1$, it was rediscovered by Gregor [9] in 1958, Lukashevich [11] in 1965, Brickman-Thomas [3] in 1977, Villarini [16] in 1992, and Christopher-Devlin [6] in 1997 (see 4], [5, 8], 10, [14] and 15] for other proofs and related topics for the case $n=1$ ). In 1998, Needham-McAllister 13 rediscovered the result for twodimensional systems via the singularity theory of C. H. Briot and J. C. Bouquet. It seems the approach in [13] applies to the arbitrary dimensional case.

It is easy to see that a necessary condition, so that the origin is an isochronous center of the system (2.1), is that all eigenvalues of $F^{\prime}(0)$ are pure imaginary with the same absolute value. The converse fails in general.

Example 1. For the system

$$
(\dot{x}, \dot{y})=\left(i x,-i y+x y^{2}\right),(x, y) \in \mathbb{C}^{2},
$$

it is easy to verify that the corresponding flow is given by

$$
\phi(t,(x, y))=\left(x e^{i t}, \frac{y e^{-i t}}{1-t x y}\right) .
$$

Clearly, the origin is not a center of the system.

\section{Proof of the Isochronous Center Theorem}

The following result is well known (see [17] for a simple proof of a more general version, where the singularity is just assumed to be isolated).

Lemma 1. If the Jacobian matrix $F^{\prime}(0)$ of $F$ at the origin is invertible, then there exist a positive number $T_{0}$ and a ball $B$ centered at the origin, such that the system (2.1) has no periodic orbit in $B^{*}=B \backslash\{0\}$ with period less than $T_{0}$.

Lemma 2. There is a ball $B \subset \Delta^{n}$ centered at the origin such that the local flow $\phi(t, z)$ of $(2.1)$ is well defined and real analytic on $[0,1] \times B$, complex holomorphic with respect to $z$,

$$
\phi([0,1] \times B) \subset \Delta^{n},
$$

and, for each real number $\tau \in[0,1]$, the Jacobian matrices $\Phi_{\tau}^{\prime}(0)$ and $F^{\prime}(0)$ of the time- $\tau$ map $\Phi_{\tau}$ and $F$ at the origin, respectively, satisfy

$$
\Phi_{\tau}^{\prime}(0)=e^{\tau F^{\prime}(0)} .
$$

The previous result is fundamental to the theory of holomorphic vector fields. The time- $\tau$ map indicates the mapping $\Phi_{\tau}: B \rightarrow \mathbb{C}^{n}$ given by

$$
\Phi_{\tau}(z)=\phi(\tau, z), z \in B,
$$


and the expression $e^{\tau F^{\prime}(0)}$ means the matrix $\sum_{k=0}^{\infty} \frac{\left(\tau F^{\prime}(0)\right)^{n}}{n !}$. If $F^{\prime}(0)=2 \pi i I$, for example, then $e^{\tau F^{\prime}(0)}=e^{2 \pi i \tau} I$, where $I$ is the unit matrix.

Proof of the Isochronous Center Theorem. Without loss of generality, assume $F^{\prime}(0)=2 \pi i I$. We shall show that the origin is an isochronous center with period 1 .

Let $B$ be a ball centered at the origin that is determined by Lemma 2 , Let $\Phi_{1}: B \rightarrow \mathbb{C}^{n}$ be the time-1 map of the local flow $\phi(t, z)$ of the system (2.1). Then by Lemma 2 we have $\Phi_{1}^{\prime}(0)=I$, which implies that the equation

$$
\Phi_{1}(z)=z+o\left(|z|^{m}\right), z \in B,
$$

holds for $m=1$. But, we can show that this equation holds for all integers $m \geq 1$ !

For any positive integer $m>1$, consider the time- $\frac{1}{m}$ map $\Phi_{\frac{1}{m}}$ of the local flow $\phi$. By Lemma 2, we have $\Phi_{\frac{1}{m}}^{\prime}(0)=e^{\frac{2 \pi i}{m}} I$, and then by the proposition, the $m$-th iteration $\Phi_{\frac{1}{m}}^{m}$ of $\Phi_{\frac{1}{m}}$ can be expressed as

$$
\Phi_{\frac{1}{m}}^{m}(z)=z+o\left(|z|^{m}\right), z \in B
$$

Thus (3.1) holds for all positive integers $m$. For

$$
\Phi_{\frac{1}{m}}^{m}(z)=\phi\left(\frac{m}{m}, z\right)=\phi(1, z)=\Phi_{1}(z), z \in B .
$$

Since $\Phi_{1}$ is a holomorphic mapping on $B$, we have proved that $\Phi_{1}=i d_{B}$, the identity mapping on $B$. Therefore, all orbits of the system that intersect $B^{*}=$ $B \backslash\{0\}$ are periodic, and then the origin is a center.

Now, let us show that the origin is, in fact, an isochronous center with period 1. We first show that for each $k \geq 2$, the origin is an isolated fixed point of the time- $\frac{1}{k}$ map $\Phi_{\frac{1}{k}}$, of the local flow. Otherwise, by the inverse function theorem, the Jacobian determinant of the mapping $z \mapsto \Phi_{\frac{1}{k}}(z)-z$ vanishes at $z=0$, and then $\Phi_{\frac{1}{k}}^{\prime}(0)$ must have an eigenvalue equal to 1 . But by Lemma 2, $\Phi_{\frac{1}{k}}^{\prime}(0)=e^{\frac{2 \pi i}{k}} I$, and then we have $k=1$. Contradiction! Thus the origin is an isolated fixed point of $\Phi_{\frac{1}{k}}$. Therefore, for an arbitrarily given integer $k_{0} \geq 2$, there exists a neighborhood of the origin in which the system has no periodic orbit of periods $\frac{1}{2}, \ldots, \frac{1}{k_{0}}$.

On the other hand, by the equation $\Phi_{1}=i d_{B}$, it is clear that the period of any periodic orbit of the system that intersects $B^{*}$ must divide 1 , and then it equals $\frac{1}{k}$ for some positive integer $k$.

Thus, there exists a punctured neighborhood of the origin in which each periodic orbit of the system either has period 1 , or has period less than $\frac{1}{k_{0}}$. Hence, by Lemma 1 and the arbitrariness of $k_{0}$, we conclude that there exists a puncture neighborhood of the origin in which all periodic orbits of the system have period 1. This completes the proof.

\section{ACKNOWLEDGMENTS}

The author would like to give thanks to Professor Meirong Zhang who told him five years ago that the normal form method is useful for iteration problems and to Professor Xiaofeng Wang who patiently taught him the fundamental result on normal forms which is used in the above proof. 


\section{REFERENCES}

[1] Arnold, V.I. Geometrical methods in the theory of ordinary differential equations, second edition, Translated by Joseph Szücs, English Translated Edited by Mark Levi, SpringerVerlag, 1988. MR0947141 (89h:58049)

[2] Arrowsmith, D. K. \& Place, C. M., An introduction to dynamical systems, Cambridge University Press, Cambridge, 1990. MR.1069752 (91g:58068)

[3] Brickman, L. \& Thomas, E. S., Conformal equivalence of analytic flows, J. Differential Equations 25 (1977), no. 3, 310-324. MR0447674 (56:5984)

[4] Chavarriga, J. \& Sabatini, M., A Survey of isochronous centers, Qualitative theory of dynamical systems, 1 (1999), 1-70. MR:1747197(2001c:34056)

[5] Cherkas, L. A., Romanovskii, V. G. \& Zołądek, H., The centre conditions for a certain cubic system, Planar nonlinear dynamical systems (Delft, 1995), Differential Equations Dynam. Systems 5 (1997), no. 3-4, 299-302. MR.1660202 (99i:34041)

[6] Christopher, C. J. \& Devlin, J., Isochronous centers in planar polynomial systems, SIAM J. Math. Anal. 28 (1997), no. 1, 162-177. MR1427732 (97k:34058)

[7] Feigenbaum, L., The center of oscillation versus the textbook writers of the early 18th century. From ancient omens to statistical mechanics, 193-202, Acta Hist. Sci. Nat. Med., 39, Univ. Lib. Copenhagen, Copenhagen, 1987. MR0961883 (90m:01015)

[8] Francoise, J.-P., Isochronous systems and perturbation theory, Journal Nonlinear Math. Phys., Vol. 12, Supplement1 (2005), 315-326. MR2117189 (2005h:34074)

[9] Gregor, J., Dynamical systems with regular hand-side, Pokroky Mat. Fys. Astronom. 3 (1958), 153-160.

[10] Hajek, O., Notes on meromorphic dynamical systems, I-III, Czechoslovak Math. J. 16 (1966), 14-40. MR0194661 (33:2870a) MR0194662(33:2870b) MR0194663(33:2870c)

[11] Lukashevich, N.A., Isochronicity of center for certain systems of differential equations, Differ. Uravn. 1 (1965), 295-302. MR0197863 (33:6023)

[12] Milnor, J., Dynamics in One Complex Variable: Introductory Lectures, Friedrick Vieweg \& Son, 2000. MR1721240(2002i:37057)

[13] Needham, D. J. \& McAllister, S., Centre families in two-dimensional complex holomorphic dynamical systems, R. Soc. Lond. Proc. Ser. A Math. Phys. Eng. Sci. 454 (1998), no. 1976, 2267-2278. MR1639872 (99d:34010)

[14] Paluszny, M., On periodic solutions of polynomial ODEs in the plane, J. Differential Equations 53 (1984), no. 1, 24-29. MR0747404 (86g:34054)

[15] Sabatini, M., Dynamics of commuting systems on two-dimensional manifolds, Ann. Mat. Pura Appl. (4) 173 (1997), 213-232. MR.1625543 (99f:34071)

[16] Villarini, M., Regularity properties of the period function near a center of a planar vector field, Nonlinear Anal. 19 (1992), no. 8, 787-803. MR1186791 (93j:34061)

[17] Zhang, G. Y., Fixed Point Indices and Invariant Periodic Sets of Holomorphic Systems, to appear in Proc. Amer. Math. Soc.

Department of Mathematical Sciences, Tsinghua University, Beijing 100084, People's Republic of China

E-mail address: gyzhang@math.tsinghua.edu.cn 